\title{
The Regulatory Network Controlling Ethanol-Induced Expression of Alcohol Dehydrogenase in the Endophyte Azoarcus sp. Strain BH72
}

\author{
Andrea Krause, ${ }^{\dagger}$ Henrike Julich, Manasee Mankar, and Barbara Reinhold-Hurek \\ Department of Microbe-Plant Interactions, Faculty of Biology/Chemistry, University of Bremen, P.O. Box 330440, 28334 \\ Bremen, Germany \\ Accepted 26 June 2017.
}

\begin{abstract}
The habitat of the nitrogen-fixing endophyte Azoarcus sp. strain BH72 is grass roots grown under waterlogged conditions that produce, under these conditions, ethanol. Strain BH72 is well equipped to metabolize ethanol, with eight alcohol dehydrogenases (ADHs), of which ExaA2 and ExaA3 are the most relevant ones. exaA2 and exaA3 cluster and are surrounded by genes encoding two-component regulatory systems (TCSs) termed ExaS-ExaR and EImS-GacA. Functional genomic analyses revealed that i) expression of the corresponding genes was induced by ethanol, ii) the genes were also expressed in the rhizoplane or even inside of rice roots, iii) both TCSs were indispensable for growth on ethanol, and iv) they were important for competitiveness during rice root colonization. Both TCSs form a hierarchically organized ethanol-responsive signal transduction cascade with ExaS-ExaR as the highest level, essential for effective expression of the ethanol oxidation system based on ExaA2. Transcript and expression levels of exaA3 increased in tcs deletion mutants, suggesting no direct influence of both TCSs on its ethanol-induced expression. In conclusion, this underscores the importance of ethanol for the endophytic lifestyle of Azoarcus sp. strain BH72 and indicates a tight regulation of the ethanol oxidation system during root colonization.
\end{abstract}

Endophytic bacteria are known to colonize the plant interior, often in high numbers, without inducing strong plant defense responses (Reinhold-Hurek and Hurek 2011). One of the best analyzed endophytes is the diazotrophic betaproteobacterium Azoarcus sp. strain BH72, originally isolated from Kallar grass (Leptochloa fusca L. Kunth) (Reinhold et al. 1986). The habitat of strain $\mathrm{BH} 72$ is the root apoplast of grasses, including rice, grown under waterlogged conditions in which the physico-chemical properties of the microenvironment are suitable for proliferation and nitrogen fixation. Although the genome sequence of strain BH72 is available (Krause et al. 2006), the molecular mechanisms relevant for an efficient beneficial interaction with its host plant are not yet well understood. Bacterial features so far known to be essential for the endophytic lifestyle are i) a type IV

Current address for H. Julich: Department of Medicine II, Saarland University Medical Center, Homburg/Saar, Germany

${ }^{\dagger}$ Corresponding author: A. Krause; E-mail: akrause@uni-bremen.de

*The $\boldsymbol{e}$-Xtra logo stands for "electronic extra" and indicates that one supplementary table is published online.

@ 2017 The American Phytopathological Society pilus-dependent adhesion to the root surface (Dörr et al. 1998), ii) a PilT- and PilX-conveyed twitching motility (Böhm et al. 2007; Shidore et al. 2012), iii) an endoglucanase EglA-facilitated entry into the root epidermis (Reinhold-Hurek et al. 2006), iv) a flagella-mediated endophytic colonization (Buschart et al. 2012), v) a type VI secretion system-regulated colonization capacity (Shidore et al. 2012), vi) an autotransporter-contributed establishment inside roots (Sarkar et al. 2017), and vii) a metabolic adaptation to the plant environment (Sarkar and ReinholdHurek 2014; Sarkar et al. 2017; Shidore et al. 2012).

One of the metabolic adaptations important for the riceendophyte interaction is the adjustment to the presence of ethanol. Rice grown in paddy fields is subjected to hypoxia and even to anoxia, which leads to fermentation provoking production and accumulation of ethanol (Agarwal and Grover 2006; Mustroph and Albrecht 2003). Azoarcus sp. strain BH72 has a strictly respiratory type of metabolism but is, nevertheless, able to grow aerobically and microarobically on ethanol as the sole source of carbon and energy (Krause et al. 2006, 2011). The genome of strain BH72 comprises eight genes encoding alcohol dehydrogenases (ADHs). Functional genomics using plasmid integration mutagenesis coupled with expression profiling, based on the plasmid-borne $g f p$ (green fluorescent protein) and gusA marker genes, revealed an ethanol-induced expression of three ADHs, of which two were also strongly expressed inside rice roots. Disruption of these two genes, encoding pyrroloquinoline quinone (PQQ)-dependent ADHs named ExaA2 and ExaA3, diminished competitiveness during endophytic colonization of rice roots. Therefore, these two ADHs are important for the endophytic lifestyle of strain BH72 (Krause et al. 2011).

For adequate adaptation to metabolic requirements in the habitat, endophytes have to react quickly by precise regulation of gene expression in response to various environmental stimuli. In order to achieve this assignment, bacteria use a variety of signal transduction systems, including two-component systems (TCSs). Components of the TCSs are histidine sensor kinases, which undergo autocatalytic phosphorylation upon an environmental stimulus, and cognate response regulators, to which the phosphoryl group is transferred, resulting in transcriptional regulation of target genes. According to P2CS, a TCS database, the genome of Azoarcus sp. strain BH72 encodes 63 histidine sensor kinases and 71 response regulators (Ortet et al. 2015). Thus, strain BH72 is well-equipped to form sophisticated signal transduction networks reacting to a wide range of environmental signals.

Until now, little is known about these signal transduction networks in Azoarcus sp. strain BH72. Here, we describe, based on functional genomics, the first steps of a hierarchical network 
controlling the metabolism of ethanol, an important carbon source in the rice-endophyte interaction.

\section{RESULTS}

\section{Chromosomal arrangement of genes involved} in ethanol oxidation.

The genome of Azoarcus sp. strain BH72 codes for eight different ADHs, two of which-the PQQ-dependent ADHs ExaA2 and ExaA3 - were shown to be of primary importance for ethanol oxidation and endophytic life with rice (Krause et al. 2011). Interestingly, exaA2 and exaA3 are situated in the same genetic locus, surrounded by genes coding for two TCSs, named ExaS-ExaR and ElmS-GacA (Fig. 1). The reading frame of exaS overlaps the one of exaR by $3 \mathrm{bp}$, indicating that these two genes are forming an operon. In contrast, elmS is separated from gacA by $213 \mathrm{bp}$. A computer-based evaluation of this intergenic region predicted a $\sigma^{70}$ promoter sequence upstream of gacA. Indeed, reverse transcription-polymerase chain reaction (RT-PCR) analysis bridging the two respective genes confirmed that they are not cotranscribed (data not shown). Apart from Azoarcus olearius DQS-4 ${ }^{\mathrm{T}}$ (Chen et al. 2013; Faoro et al. 2017), the closest relative of Azoarcus sp. strain $\mathrm{BH} 72$, none of the so-farsequenced bacteria has a similar arrangement of genes involved in ethanol oxidation.

The response regulators (ExaR, GacA) carry a helix-turnhelix DNA-binding domain of the LuxR type, indicating that they serve as transcriptional activators. They have an overall sequence identity to each other of $45 \%$. ExaS and ElmS are classical histidine sensor kinases with a HAMP domain, a domain known to be part of integral sensor kinases and to be involved in transmission of periplasmic signals to the cytoplasma (Aravind and Ponting 1999). In fact, the prediction of two transmembrane helices in ExaS and ElmS indicates a membrane-bound localization with a periplasmic loop. Their overall sequence identity is only $28 \%$. Both TCSs were found to have significant sequence identity of up to $44 \%$ to EraR-EraS of Pseudomonas aeruginosa, a TCS regulating expression of the PQQ-dependent ADH ExaA (Schobert and Görisch 2001). Taking the genomic location and the sequence identity in consideration, a potential role of ExaS-ExaR and ElmS-GacA in regulation of the $\mathrm{ADH}$ expression in Azoarcus sp. strain $\mathrm{BH} 72$ could be postulated.

\section{Growth of putative regulatory mutants on ethanol.}

In order to clarify the role of the two TCSs in the ethanol metabolism, mutants in each TCS were constructed. According to the predicted operon structure mutants were separately generated in gacA and elmS through in-frame deletion, while exaRS were inactivated together by a combination of deletion and insertion of the streptomycin- and spectinomycin-resistant $\left(\mathrm{Sm}^{\mathrm{r}} / \mathrm{Sp}^{\mathrm{r}}\right)$ antibiotic resistance cassette (Fig. 1A). Growth behavior of the mutant strains was determined on malate- and ethanol-containing media, both being major carbon sources for Azoarcus sp. strain BH72 (Reinhold-Hurek et al. 1993). Inactivation of these genes did not affect cultivation on malate but prevented the mutants growth on ethanol (Fig. 2A). As mutant BHexaRSs might show a possible polar effect on azo2969-the gene directly downstream of exaR - the plasmid integration mutant BHazo2969tg was designed (Fig. 1A). Growth of this mutant strain on ethanol-containing media was with a growth rate of $0.40 \pm 0.04$, not significantly reduced in comparison with the wild type. Therefore, the lack of growth on ethanol could clearly be assigned to the absence of the TCS ExaS-ExaR.

Interestingly, the observed growth phenotype of all TCSs mutant strains was more severe than the one obtained by disruption of exaA2 or exaA3, which still showed 19 or $57 \%$ of the wild type growth on ethanol (Krause et al. 2011), and even more than the one determined for a exaA2-exaA3 double mutant
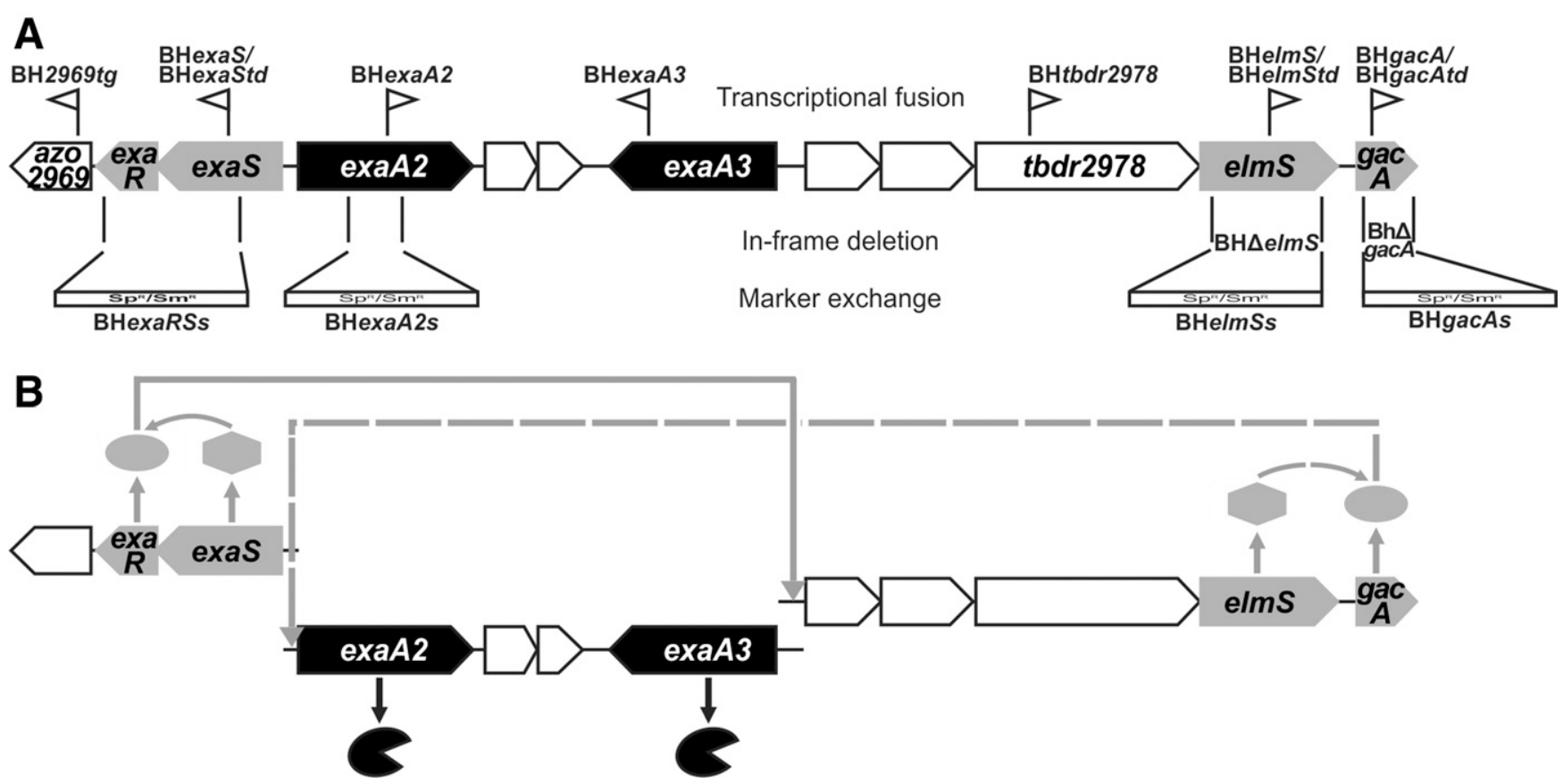

Fig. 1. The exaA2-exaA3 gene cluster. Structural genes encoding pyrrolo-quinoline quinone-dependent alcohol dehydrogenases are shown in black while genes coding for two-component regulatory systems are marked in gray. $1 \mathrm{~cm}=1,000 \mathrm{bp}$. A, Mutants constructed in this study. Positions of transcriptional fusions to gfp-gusA, tdTomato-gusA, or tdTomato are labeled by triangles and are indicated by the corresponding names. Areas deleted in in-frame deletion mutants or replaced by the streptomycin- and spectinomycin-resistant antibiotic resistance cassette in marker exchange mutants are displayed. All mutant names are given. B, The ethanol-responsive signal transduction pathway forming a hypothetical hierarchical network controlling exaA2 expression. Response regulators are illustrated by circles. Hexagons display sensor kinases. 
with a growth rate of $0.05 \pm 0.013$. Therefore, in Azoarcus sp. strain BH72 both TCSs are essential for the ethanol utilization as carbon source.

\section{Expression profile of the sensor kinases ExaS and EImS and the response regulator GacA in free-living cells.}

Transcriptional fusions of the TCSs encoding operons with the reporter gene gusA were constructed to analyze their expression levels under various conditions (Fig. 1A). In Azoarcus sp. strain $\mathrm{BH} 72$, the expression of exaS, elmS, and gacA was significantly enhanced by the addition of ethanol, as carbon source, to the growth medium versus malate (Fig. 2B), indicating that these genes are members of the ethanol-induced regulon. This ethanol-regulated induction was independent of aerobic or nitrogen-fixing microaerobic growth conditions and varied between factors of three to ten based on the gene and the condition analyzed. While the overall expression of $\operatorname{elmS}$ and gacA did not differ between aerobic and nitrogen-fixing microaerobic conditions, exaS showed a significantly higher expression in the nitrogen-fixing microaerobic environment compared with the aerobic one (defined by the $t$ test, $P<0.001$ ). This twofold increased expression was also detectable in N-replete, microaerobic, ethanol-containing growth media with 1,805 \pm 254 Miller units, suggesting that exaS not only belongs to the ethanolinduced regulon but, also, overlaps with the oxygen-responsive regulon.

\section{ExaRS, GacA, and ElmS are involved in induction} of expression by ethanol of exaA2 but not of exaA3.

The ethanol-induced expression of exaS, elmS, and gacA as well as the sequence identity to EraR-EraS of $P$. aeruginosa (Schobert and Görisch 2001) evoke the hypothesis that these two TCSs of strain BH72 are also involved in the ethanolresponsive signal cascade. Endpoints of this signal cascade are presumably the ADH-encoding genes exaA2 and exaA3, which showed drastically increased expression by the addition of ethanol to the culture media in comparison with malatecontaining media (Krause et al. 2011). Therefore, the ethanolinduced expression of exaA2 and exaA3 was studied in the TCS deletion mutant strains (Fig. 1A). Using chromosomally integrated transcriptional fusions of both genes to gusA as well as RT-PCR analyses revealed an alteration in gene expression in the presence of ethanol (Fig. 3A and B). Expression of exaA2 was lower or noninduced in all generated putative regulatory mutants, as compared with the wild type. Interestingly, neither was $\beta$-glucuronidase activity detectable nor were transcripts of exaA2 visible in the mutant, where the TCS ExaR-ExaS was inactivated. Deletion of elms or gacA lowered exaA2-driven $\beta$-glucuronidase activity to 20 and $30 \%$, respectively, of the wildtype level. This reduced expression in the presence of ethanol was also seen for the exaA2 transcript levels in the respective mutant strains. Thus, both TCSs are required for effective, ethanol-induced expression of exaA2.

On the contrary, the ethanol-induced exaA3 expression, as determined by $\beta$-glucuronidase activity, was significantly elevated by a factor of 1.8 in the exaRS and elmS deletion mutants, in comparison with the wild type. Equally, exaA3 transcript levels increased drastically. When the response regulator GacA was inactivated, no significant changes in exaA3 promoter activity nor in exaA3 transcript levels could be observed (Fig. 3A and B). This observed elevated induction of exaA3 expression by ethanol in the mutant strains raised the question whether the absence of ExaA2 was compensated for by a more severe induction of exaA3 expression through ethanol. In order to verify this assumption, the exaA3-gusA transcriptional fusion was integrated into the genome of $\mathrm{BHexaA2s}$, a mutant strain where part of exaA2 was replaced by a $\mathrm{Sm}^{\mathrm{r}} / \mathrm{Sp}^{\mathrm{r}}$ antibiotic resistance cassette (Krause et al. 2011). As exaA3 expression in this mutant strain was with $3,782 \pm 775$ Miller units identical to the one in the wild type, it can be stated that the increased expression of exaA3 is not directly linked to an absence of ExaA2. The inability of ethanol consumption in the tcs deletion mutants seems to be adjusted at another step in the metabolism and is then transmitted from there by one or more unknown regulators towards exaA3.

\section{ExaRS, ElmS, and GacA forming}

a hierarchical ethanol-responsive signal cascade.

Reporter gene fusions of exaS, elmS, or gacA integrated into the genomes of the different TCS deletion mutant strains allowed the determination of the mutual interference with each other on their expression. The results showed that exaS expression does not depend on the presence of the TCS ElmSGacA (Fig. 3C). Also, expression of gacA appears to be independent of the TCS ExaS-ExaR as well as the sensor kinase ElmS. On the contrary, the sensor kinase ExaS and the response regulator ExaR are required for controlling the ethanol-induced expression of elmS. Therefore, the TCS ExaS-ExaR seems to represent a higher level of regulatory components in the ethanol-responsive signal cascade than the TCS ElmS-GacA (Fig. 1B).
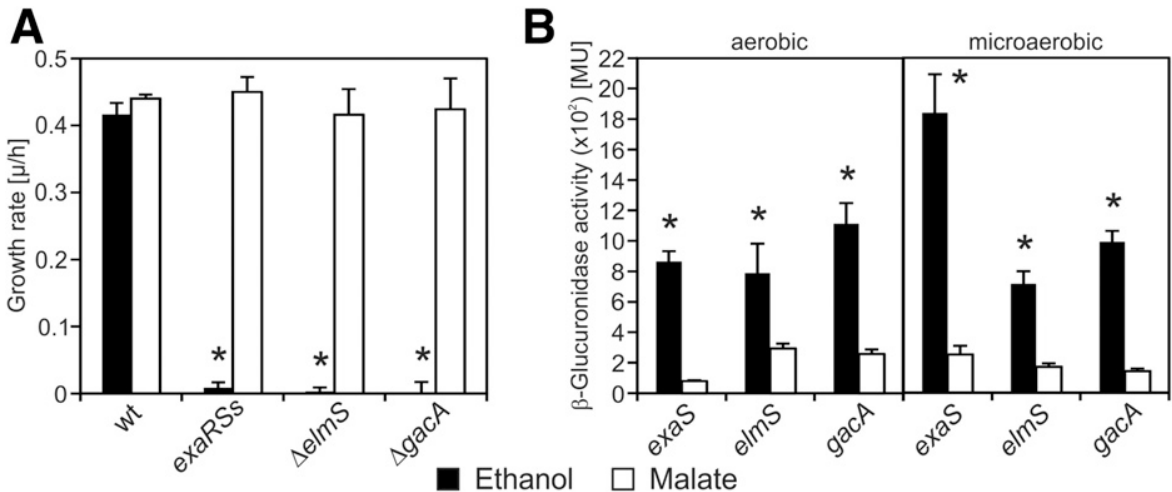

Fig. 2. Analyses of strains carrying mutations in genes encoding two-component regulatory systems. Strains were cultured in synthetic medium (SM) under aerobic conditions or in a nitrogen-free SM with a microaerobic environment, both supplemented with ethanol (black columns) or malate (white columns). A, Growth of free-living Azoarcus strains carrying deletion of the desired gene. Growth rates (average \pm standard deviation) were determined from at least four independent cultures. Asterisks show significant differences between mutants versus wild type $(P<0.001)$. B, Promoter activity of the desired genes transcriptionally fused to gusA during the free-living stage. Activity is given as $\beta$-glucuronidase activity in Miller units (average \pm standard deviation) determined from five independent cultures measured in duplicate. Asterisks indicate significantly higher $\beta$-glucuronidase activity in ethanol-containing medium compared with malate medium $(P<0.001)$. 
A gene, $t b d r 2978$, located upstream of $\operatorname{elmS}$ (Fig. 1A), is cotranscribed with it. Expression analyses of this gene revealed that the ethanol-induced expression is not altered in the elmS deletion mutant compared with the one observed in the wild type
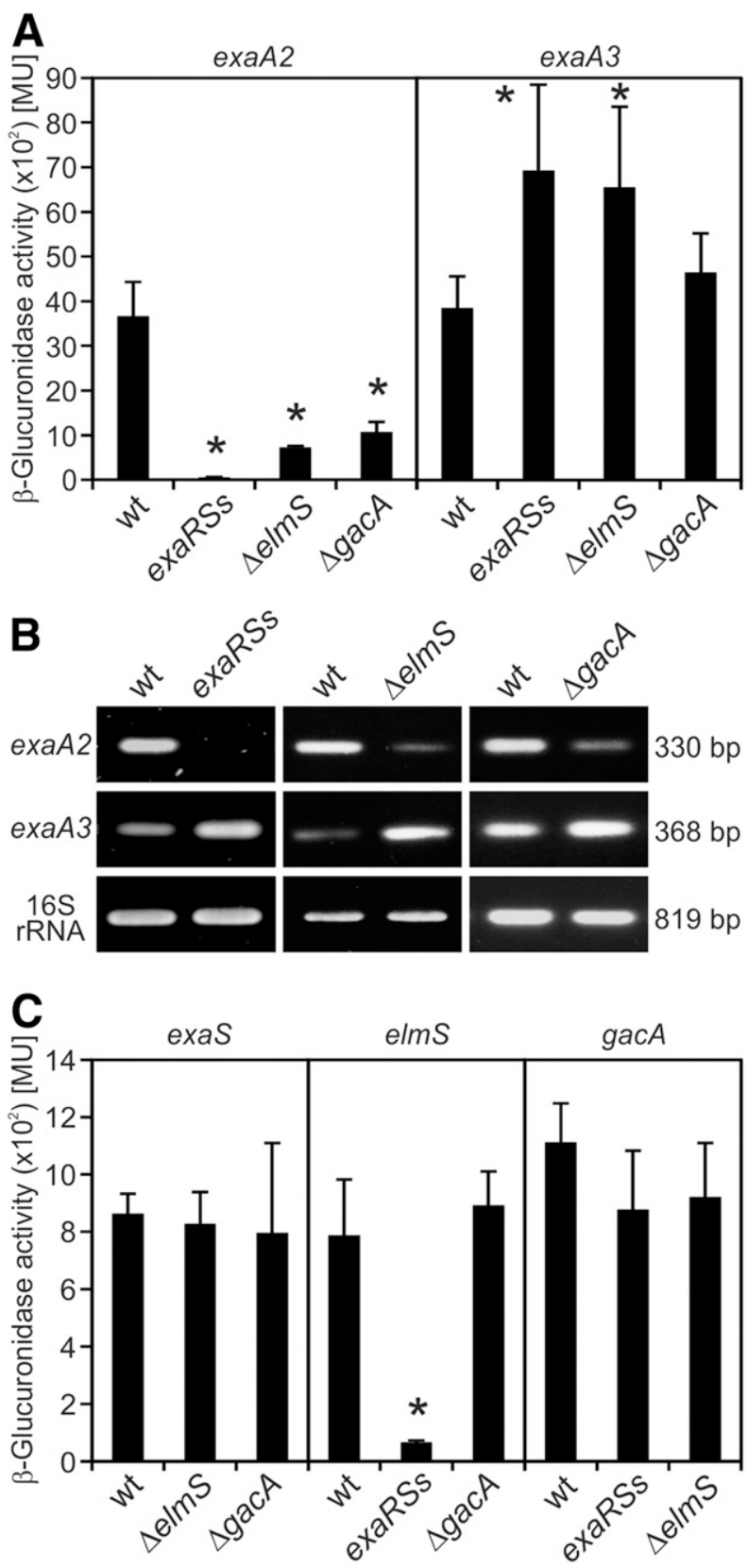

Fig. 3. Influence of the two-component regulatory systems on the expression of genes important for ethanol oxidation. For determination of promoter activities, the $\beta$-glucuronidase activity test was used after $6 \mathrm{~h}$ of growth under aerobic conditions in synthetic medium containing ethanol. Activity is specified as Miller units (average \pm standard deviation) calculated from five independent cultures measured in duplicate. Significantly different activities in comparison with the activity in the wild type are indicated by asterisks $(P<$ $0.001)$. For determination of transcript levels semiquantitative reverse transcription-polymerase chain reaction analyses were performed on total RNA extracted from aerobically grown overnight cultures in Voll medium containing ethanol. A, Promoter activity of the alcohol dehydrogenase-encoding genes exaA2 and exaA3 in wild type and different two-component regulatory system (TCS) mutant strains. B, Transcript levels of exaA2 and exaA3 in wild type and different TCS mutant strains. Representative results of cycle 26 or 28 are shown. Corresponding controls without reverse transcriptase showed no presence of DNA in the total RNA samples (data not shown). C, Expression profile of exaS, elmS, and gacA in different TCS mutant strains. (data not shown). Apparently, the sensor kinase ElmS is not essential for its own expression.

\section{Analysis of importance of ExaS-ExaR and EImS-GacA for rice root colonization.}

ExaA2 conveyed a competitive advantage to Azoarcus sp. strain BH72 during endophytic colonization (Krause et al. 2011). The participation of the TCSs ExaS-ExaR and ElmSGacA in controlling the exaA2 expression raised the question of the significance of these systems for rice root colonization. To study this question, marker exchange mutants of $\operatorname{elmS}$ and $\mathrm{gacA}$ using the $\mathrm{Sm}^{\mathrm{r}} / \mathrm{Sp}^{\mathrm{r}}$ antibiotic resistance cassette were constructed, based on the in-frame deletion mutants (Fig. 1A). For exaS, the mutant described above was used. In a competitive approach, roots of rice seedlings were inoculated by a mixture of equal amounts of Azoarcus sp. strain BH72 and one mutant strain. The result disclosed that the wild type significantly colonized the roots, by a factor of 2.2 , better than any of the mutant strains (Table 1). This factor was identical to the one achieved when the ADH-encoding genes exaA2 or exaA3 were deleted (Krause et al. 2011). Therefore, these two TCSs are as important for effective root colonization as the $\mathrm{ADHs}$ themselves, most probably by also regulating in planta the gene expression of exaA2.

This assumption implies that the two TCSs are expressed in planta. In order to verify the expression, transcriptional fusions of exaS, elmS, and gacA to tdTomato were constructed, identical to the previously described strategy of $g f p$ - $g u s A$ fusions (Fig. 1A). TdTomato was chosen as a fluorescent marker on the basis of its exceptional brightness, as expression of the tcs in free-living strain BH72 was insufficiently high (Fig. 2B) for GFP-based single-cell microscopic visibility (data not shown). As seen by the red fluorescence signal, all three bacterial genes were expressed during rice root colonization (Fig. 4). Expression of exaS was detectable at the rhizoplane and at the first layer of the endorhizosphere in which the expression of elmS and gacA was also observable. Therefore, the different microhabitats of a plant-microbe interaction, the root interior as well as the interface between the root and the rhizosphere, provide suitable conditions for the expression of the two tcs systems, which then might lead in planta to the activation of exaA2 expression.

\section{DISCUSSION}

Azoarcus sp. strain BH72 is metabolically not very versatile. As an adaption to the plant-compatible endophytic lifestyle, energy can be mainly obtained by oxidation of dicarboxylic acids or ethanol (Reinhold-Hurek et al. 1993). Having eight ADHs, of which the PQQ-dependent ADHs ExaA2 and ExaA3 are the most important ones for growth on ethanol, strain $\mathrm{BH} 72$ seems to be well-prepared for ethanol metabolism (Krause et al. 2011).

The close genomic vicinity of exaA2 and exaA3 is not unique to Azoarcus sp. strain BH72. This was also found in different Pseudomonas strains (Tribelli et al. 2015), which are able to grow on ethanol as carbon source as well. In strain $\mathrm{BH} 72$, adjacent to the exaA2-exaA3 cluster, are four genes coding for

Table 1. Bacterial competition during endophytic root colonization

\begin{tabular}{lcc}
\hline $\begin{array}{l}\text { Strain in competition } \\
\text { with BH72 }\end{array}$ & $\begin{array}{c}\text { Colonizing bacteria } \\
(\text { in } \%)^{\mathbf{a}}\end{array}$ & $\boldsymbol{P}$ value \\
\hline BHexaRSs & $30.7 \pm 5.4$ & $9.4 \times 10^{-4}$ \\
BHelmSs & $31.5 \pm 2.5$ & $5.7 \times 10^{-5}$ \\
BHgacAs & $31.3 \pm 1.2$ & $3.2 \times 10^{-6}$ \\
\hline
\end{tabular}

a Analyses based on three independent experiments with a total of 33 plants per mixture. Shown is the average \pm standard deviation. 
two localized TCSs. Both TCSs were shown to be essential for the ethanol-responsive signal transduction pathway regulating effective expression of exaA2. Surprisingly, none of the TCSs were involved in regulation of ethanol-induced expression of exaA3, although these two ADHs have the same substrate specificity (Krause et al. 2011).

The organization of the TCSs in this signal transduction pathway is hierarchical (Fig. 1B), encompassing, until now, two levels, similar to the regulatory networks of the ethanol oxidation system of $P$. aeruginosa (Gliese et al. 2004; Mern et al. 2010) and P. putida (Promden et al. 2009) as well as of the methanol oxidation system of Methylobacterium extorquens (Springer et al. 1997). The highest level in this hypothetical signal cascade is the TCS ExaS-ExaR, which is important for the ethanol-induced expression of elmS. Then ElmS together with GacA, whose ethanol-induced expression is independent of ExaS-ExaR, directly or indirectly regulates the exaA2 expression in response to the availability of ethanol.

The proposed hierarchical network is based on the assumption that the sensor kinases ExaS and ElmS form TCSs with their cognate response regulators ExaR and GacA, respectively, without functional interchange between each other. This nonfunctional interchange is experimentally supported by the inability of the mutant strains to grow on ethanol. On the other hand, the proposed hierarchical network fails to explain the observed inability of all regulatory mutants to grow on ethanol as sole carbon source. As the regulatory network does not influence the ethanol-induced expression of exaA3, the ethanol metabolism should proceed. Deletion of any element of the network even increased expression of exaA3. Therefore, it must be postulated that the network also regulates expression of additional genes coding for components of the ethanol oxidation system, similar to what is seen in P. aeruginosa (Gliese et al. 2004).

The ethanol-induced expression of exaS, elmS, and gacA was also observed under microaerobic conditions, in which Azoarcus sp. strain BH72 is capable of fixing nitrogen (Hurek et al. 1987). Expression of these genes was also detectable in the rhizoplane or even inside rice roots. Additionally, disruption of one of the genes reduced the competitiveness in colonizing rice roots to the same extent as the exaA2 deletion mutant (Krause et al. 2011). Therefore, it could be hypothesized that the proposed regulatory network is also regulating in planta the gene expression of exaA2. Furthermore, the ethanol-triggered expression of the four $t c s$ genes indicates that ethanol is most likely provided by the plant partner of this endophytic interaction. This assumption is corroborated by the results on the exaA2 and exaA3 expression as previously described by Krause et al. (2011). Thus, ethanol is an important carbon source for the endophytic life of Azoarcus sp. strain BH72.

\section{MATERIALS AND METHODS}

\section{Bacterial strains and conditions of cultivation.}

Bacterial strains used are listed in Supplementary Table S1. Escherichia coli was cultivated at $37^{\circ} \mathrm{C}$ in Luria-Bertani medium containing suitable antibiotics (Sambrook et al. 1989), while Azoarcus sp. strains were generally grown at $37^{\circ} \mathrm{C}$ in the complex Voll medium (VM) (carbon source as shown below, $3 \mathrm{~g}$ of bacto pepton, $1 \mathrm{~g}$ of yeast extract, $1.1 \mathrm{~g}$ of $\mathrm{NaCl}, 0.5 \mathrm{~g}$ of $\mathrm{NH}_{4} \mathrm{Cl}, 0.6 \mathrm{~g}$ of $\mathrm{K}_{2} \mathrm{HPO}_{4}, 0.4 \mathrm{~g}$ of $\mathrm{KH}_{2} \mathrm{PO}_{4}, 0.2 \mathrm{~g}$ of $\mathrm{MgSO}_{4}, 66 \mathrm{mg}$ of $\mathrm{Fe}(\mathrm{III})-$ EDTA, $26 \mathrm{mg}$ of $\mathrm{CaCl}_{2} \cdot 2 \mathrm{H}_{2} \mathrm{O}, 10 \mathrm{mg}$ of $\mathrm{MnSO}_{4} \cdot \mathrm{H}_{2} \mathrm{O}, 2 \mathrm{mg}$ of $\mathrm{Na}_{2} \mathrm{MoO}_{4} \cdot 2 \mathrm{H}_{2} \mathrm{O}, \mathrm{pH}$ 6.8). In addition, the Azoarcus strains were cultured aerobically in the synthetic medium (SM) (carbon source as shown below, $0.5 \mathrm{~g}$ of $\mathrm{NH}_{4} \mathrm{Cl}, 0.6 \mathrm{~g}$ of $\mathrm{K}_{2} \mathrm{HPO}_{4}, 0.4 \mathrm{~g}$ of $\mathrm{KH}_{2} \mathrm{PO}_{4}, 0.2 \mathrm{~g}$ of $\mathrm{MgSO}_{4}, 0.1 \mathrm{~g}$ of NaCl, $66 \mathrm{mg}$ of $\mathrm{Fe}(\mathrm{III})$-EDTA, $26 \mathrm{mg}$ of $\mathrm{CaCl}_{2} \cdot 2 \mathrm{H}_{2} \mathrm{O}, 10 \mathrm{mg}$ of $\mathrm{MnSO}_{4} \cdot \mathrm{H}_{2} \mathrm{O}, 2 \mathrm{mg}$ of $\mathrm{Na}_{2} \mathrm{MoO}_{4} \cdot 2 \mathrm{H}_{2} \mathrm{O}, \mathrm{pH}$ 6.8). Nitrogen-fixing conditions were achieved by using SM devoid of nitrogen in rubber stopper-sealed Erlenmeyer flasks with microaerobic conditions of an initial $1.2 \%$ $\mathrm{O}_{2}$ in the $\mathrm{N}_{2}$ headspace (Krause et al. 2011). All media were supplemented either with ethanol $(6 \mathrm{ml} / \mathrm{liter})$ or malic acid $(5$ $\mathrm{g} / \mathrm{liter})$ as carbon source. Antibiotics used were kanamycin $(\mathrm{Km})$ or Sp at a final concentration of 30 and $40 \mu \mathrm{g} / \mathrm{ml}$, respectively.

\section{Nucleic acid manipulation and sequence data analysis.}

Standard molecular DNA techniques were carried out as described by Sambrook et al. (1989). Sequences were determined

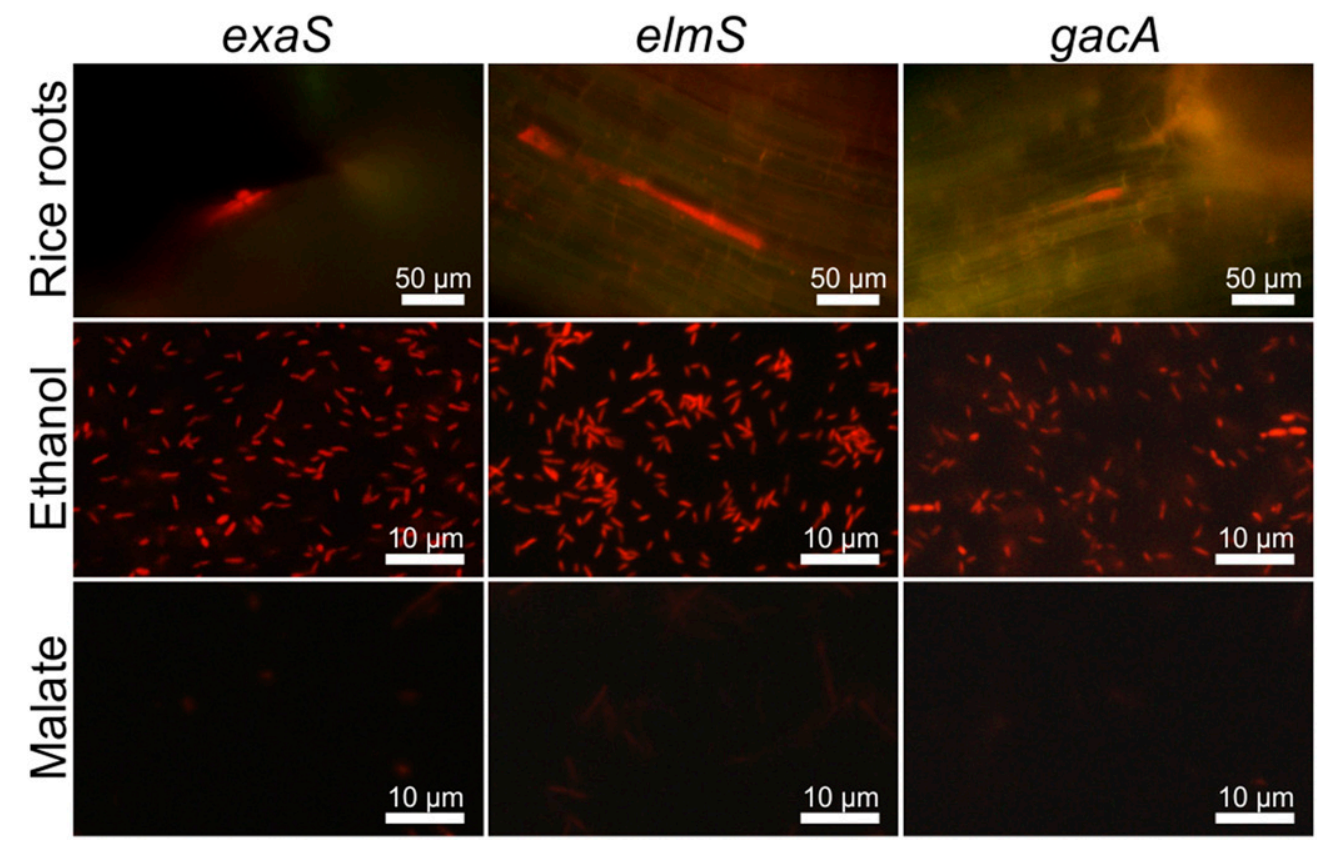

Fig. 4. Detection of bacterial gene expression in the plant-rhizophere interface. Rice seedlings were inoculated with BHexaStd, BHelmStd, or BHgacAtd and were cultivated gnotobiotically in a growth chamber for 14 days. Bacterial cultures were grown overnight in Voll medium supplemented with ethanol or malate as carbon source. Representative photographs are shown. Settings of the fluorescence microscope and the video camera were equal for all micrographs of roots. Also, for bacterial cultures, all settings were identical. 
by the traditional Sanger method (LGC Genomics, Berlin). Total RNA isolation from 20 to $50 \mathrm{ml}$ of overnight cultures grown in complex media with the addition of ethanol were performed using the hot phenol extraction methods as described in more detail by Hauberg-Lotte et al. (2012). Genomic DNA contaminations in the RNA preparation were removed, either by treatment with DNase I (Roche Diagnostics GmbH, Mannheim, Germany) including the RNase inhibitor Superase (Life Technologies, Darmstadt, Germany,) according to manufacturer's instructions and subsequent phenol-chloroform-isoamylalcohol extraction followed by a precipitation step, or by applying the RNeasy Plus Mini Kit (Qiagen, Hilden, Germany). Finally, RNA samples were stored in $1 \times$ RNAsecure (Life Technologies, Darmstadt, Germany).

Analyses of sequences were performed as follows: comparison through the BLAST program (Altschul et al. 1997) of the National Center for Biotechnology Information (NCBI), identification of protein domains through the conserved domain database at NCBI (Marchler-Bauer et al. 2011), the Pfam database of the Sanger Institute (Finn et al. 2014), or TIGRFAM of the J. Craig Venter Institute (Haft et al. 2013), recognition of signal peptides and transmembrane helices through SignalP 3.0 (Bendtsen et al. 2004) or TMHMM 2.0 (Krogh et al. 2001) of the Center for Biological Sequence Analysis (Aarhus, Denmark), and prediction of $\sigma^{70}$ promoters through BPROM of Softberry, Inc. (Mount Kisco, NY, U.S.A.). Synteny of the exaA2-exaA3 gene cluster in microbial genomes was analyzed with SyntTax (Oberto 2013) and MultiGeneBlast (Medema et al. 2013).

\section{Construction of mutant strains.}

elmS and gacA were disrupted either by in-frame deletion or marker exchange mutagenesis. For this, both flanking regions of each gene were amplified using the $P f u$ DNA polymerase (Thermo Fisher Scientific, Sankt Leon-Rot, Germany). The PCR fragments contained $128 \mathrm{bp}$ of the $5^{\prime}$ end and $163 \mathrm{bp}$ of the $3^{\prime}$ end of elmS or, for gacA, 71 and $34 \mathrm{bp}$, respectively. These fragments were cloned into pUC18 or pJET1.2/blunt (Thermo Fisher Scientific) and were subsequently verified by sequencing (LGC Genomics). Both flanking regions of one gene were joined together and were cloned into the mobilizable vector pK18mobsacB (Schäfer et al. 1994). The corresponding plasmids were transferred into Azoarcus sp. strain BH72 by biparental conjugation. In a first step, plasmid integration into the chromosome was monitored by the vector-based $\mathrm{Km}^{\mathrm{r}}$. One of these transconjugants was cultivated and, finally, double crossover events were identified by sucrose selection and Km sensitivity. Accuracy of the deletion was then verified by PCR. In order to create the marker exchange mutants, the recombinant $\mathrm{pK} 18 \mathrm{mobsacB}$ plasmids carrying the two flanking regions were used. The antibiotic resistance cassette $\mathrm{Sm}^{\mathrm{r}} / \mathrm{Sp}^{\mathrm{r}}$ obtained

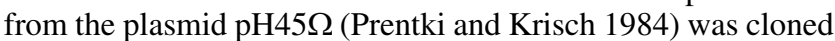
into the junction between these two regions. After biparental conjugation into Azoarcus sp. strain BH72, selection of double crossovers was performed by the Sm resistance and $\mathrm{Km}$ sensitivity. The DNA segment of exaRS to be deleted covered 911 bp of the $3^{\prime}$ end of exaS as well as 558 bp of the $5^{\prime}$ end of exaR. The deleted fragment was replaced by the $\mathrm{Sm}^{\mathrm{r}} / \mathrm{Sp}^{\mathrm{r}}$ antibiotic resistance cassette. Suitable DNA fragments around exaRS were amplified using the Pfu DNA polymerase, followed by cloning into the pK18mob2 (Kirchner and Tauch 2003), verification by sequencing, insertion of the resistant cassette, and conjugation into Azoarcus sp. strain BH72. Double crossover events at homologous sites were selected as described above.

To generate transcriptional fusions to gfp-gusA, tdTomato, or tdTomato-gusA, appropriate regions of the $5^{\prime}$ end of the desired genes were amplified with DreamTaq DNA polymerase
(Thermo Fisher Scientific). Thereafter, the PCR products were cloned into vectors pK18GGST (Krause et al. 2011), pK18TST (Sarkar et al. 2017), or pK18TGST, the latter two derivatives of pK18GGST in which the $g f p$-gusA cassette was replaced by the promoterless tdTomato or by the promoterless tdTomato-gusA marker genes. Transfer of the obtained plasmids into Azoarcus sp. strain $\mathrm{BH} 72$ and its in-frame deletion mutants was performed via biparental conjugation followed by selection of the plasmid integration into the chromosome through vector-based $\mathrm{Km}^{\mathrm{r}}$. Correct integration events at homologous sites of all mutant strains were confirmed by PCR and Southern blot analysis.

\section{Bacterial growth analyses in culture.}

The growth of Azoarcus sp. strain BH72 and its mutants was determined essentially as described by Krause et al. (2011). For that, strains were precultured in complex VM with ethanol or malate for at least $13 \mathrm{~h}$ at $37^{\circ} \mathrm{C}$. After centrifugation, cells were washed two times and, finally, were adjusted to an optical density at $600 \mathrm{~nm}\left(\mathrm{OD}_{600 \mathrm{~nm}}\right)$ of 0.05 in SM supplemented either with ethanol or malate. Incubation of the cultures took place at $37^{\circ} \mathrm{C}$ until stationary phase was reached, during which the $\mathrm{OD}_{600 \mathrm{~nm}}$ was determined at different time points. Growth rates were calculated for each culture separately, were averaged, and then, were statistically examined by a two-tailed $t$ test.

\section{Quantification of gene expression using the $\beta$-glucuronidase activity.}

Cells of overnight cultures grown at $37^{\circ} \mathrm{C}$ in complex VM with malate were collected by centrifugation and were washed two times with nitrogen-free SM containing no carbon source. Finally, they were resuspended in SM for aerobic growth or in nitrogen-free SM for microaerobic growth comprising ethanol or malate as carbon source. Depending on their growth capability in the medium used, cultures were adjusted to an $\mathrm{OD}_{600 \mathrm{~nm}}$ of 0.1 or 0.3 and were then incubated for $6 \mathrm{~h}$ under aerobic or nitrogen-fixing microaerobic conditions at $37^{\circ} \mathrm{C}$. Activity of the $\beta$-glucuronidase was assayed quantitatively, as described by Jefferson et al. (1986) with modifications (Krause et al. 2011), and was expressed in Miller units. The average of each culture was calculated and the significance was determined through the two-tailed $t$ test.

\section{Analyses of gene expression using RT-PCR.}

Semiquantitative RT-PCR was performed from isolated total RNA in a two-step system, according to manufacturer's protocols. In the first step, $80 \mathrm{ng}$ of RNA was applied to cDNA synthesis at $60^{\circ} \mathrm{C}$ for 30 min with gene-specific reversed primers, using the RevertAid Premium reverse transcriptase (Thermo Fisher Scientific). Thereafter, in the second step, PCR amplification of exaA2 or exaA3 with the gene-specific primer sets exaA2RT-for (5' CCTCCATCGTCGCGCTCGAC 3') and exaA2RT-rev (5' ACCTTGCCGGTGGGGGAGTT $3^{\prime}$ ) or exaA3RT-for (5' GGCCAGGAATCGCAACCGCT 3') and exaA3RT-rev (5' TTCCATGCGGCCGACCACAC $3^{\prime}$ ) was carried out. For this, $2 \mu \mathrm{l}$ of the cDNA was amplified using the DreamTaq DNA polymerase (Thermo Fisher Scientific) in 30 cycles of $1 \mathrm{~min}$ at $95^{\circ} \mathrm{C}, 0.5 \mathrm{~min}$ at $68.5^{\circ} \mathrm{C}$, and $1 \mathrm{~min}$ at $72^{\circ} \mathrm{C}$. $16 \mathrm{~S}$ rRNA was utilized to ensure that equal amounts of RNA were applied. For this, only 20 ng of RNA was inserted in the cDNA synthesis. The gene-specific primer pairs were either TH3 and TH5 (Hurek et al. 1993) or 799 f (Chelius and Triplett 2001) and 1492r (Lane 1991). PCR amplification was performed for 30 cycles at $95^{\circ} \mathrm{C}$ for $1 \mathrm{~min}, 70^{\circ} \mathrm{C}$ for $1 \mathrm{~min}$ (TH3-TH5) or $50^{\circ} \mathrm{C}$ for $30 \mathrm{~s}(799 \mathrm{f}-1492 \mathrm{r})$, and $72^{\circ} \mathrm{C}$ for $1 \mathrm{~min}$. Lack of DNA in the RNA preparation was confirmed by PCR without previous cDNA synthesis. Aliquots $(5 \mu \mathrm{l})$ were taken at different cycles and were subsequently analyzed on $1.8 \%$ agarose gels. 


\section{Evaluation of Oryza sativa root colonization.}

Caryopses of Oryza sativa subsp. indica cv. IR36 obtained from the International Rice Research Institute (Los Banos, The Philippines) were dehusked, surface sterilized, and germinated as described earlier (Hurek et al. 1994; Krause et al. 2011), with the modification that germination was performed for 3 days in darkness at $30^{\circ} \mathrm{C}$, followed by 1 day at $23^{\circ} \mathrm{C}$ with a daytime of $10 \mathrm{~h}$ and a light intensity of approximately $100 \mu \mathrm{mol}$ photons $\mathrm{m}^{-2} \mathrm{~s}^{-1}$. Bacterial strains were grown overnight in $\mathrm{SM}$ at $37^{\circ} \mathrm{C}$, followed by two centrifugation steps with a final resuspension in ethanol-free plant medium $\left(0.6 \mathrm{~g}\right.$ of $\mathrm{K}_{2} \mathrm{HPO}_{4}, 0.4 \mathrm{~g}$ of $\mathrm{KH}_{2} \mathrm{PO}_{4}, 200 \mathrm{mg}$ of $\mathrm{MgCl}_{2} \cdot 6 \mathrm{H}_{2} \mathrm{O}, 100 \mathrm{mg}$ of $\mathrm{NaCl}, 26 \mathrm{mg}$ of $\mathrm{CaCl}_{2} \cdot 2 \mathrm{H}_{2} \mathrm{O}, 20 \mathrm{mg}$ of DL-malic acid, $13 \mathrm{mg}$ of $\mathrm{Fe}$ (III)-citrate hydrate, $12 \mathrm{mg}$ of $\mathrm{MnSO}_{4} \cdot \mathrm{H}_{2} \mathrm{O}, 4 \mathrm{mg}$ of $\mathrm{Na}_{2} \mathrm{MoO}_{4} \cdot 2 \mathrm{H}_{2} \mathrm{O}, 3 \mathrm{mg}$ of $\mathrm{H}_{3} \mathrm{BO}_{3}, 0.2 \mathrm{mg}$ of $\mathrm{ZnSO}_{4} \cdot 7 \mathrm{H}_{2} \mathrm{O}, 0.1 \mathrm{mg}$ of $\mathrm{CuSO}_{4} \cdot 5 \mathrm{H}_{2} \mathrm{O}$, pH 6.8 [Egener et al. 1999]), and $2 \times 10^{8}$ cells were added to the germinated rice seedlings. For the competitive approach, the applied bacterial cells constituted a 1:1 mixture of the wild type and a mutant strain, while, for in planta expression analyses, only single strains were used. In order to verify the accuracy of the 1:1 mixture, dilutions were plated on SM. Then, randomly, 100 colonies were transferred in parallel to VM-ethanol plates, with and without appropriate antibiotics to distinguish the mutant strain from the wild type. Gnotobiotic cultivation of plants under conditions devoid of the addition of external ethanol and quantification of endophytic colonization and competitiveness of mutant strains versus wild type were carried out as described by Krause et al. (2011). Results were statistically analyzed by the two-tailed $t$ test.

Before monitoring of tdTomato expression, root samples were incubated for $1 \mathrm{~h}$ at $4^{\circ} \mathrm{C}$. Visualization of tdTomato fluorescence was done through the Zeiss Axioplan 2 microscope (Carl Zeiss AG, Jena, Germany), with an excitation filter BP 550/25, a beam splitter FT 570, and an emission filter BP 605/70 (Filter set $43 \mathrm{HE}$ ). The filter set 09 (excitation filter BP 450-490, beam splitter FT 510, and emission filter LP 515) was used to monitor root autofluorescence. Micrographs were recorded at identical settings, for comparative pictures, with the Axiocam 503 color camera (Carl Zeiss AG) for both filter sets, and finally, were overlaid.

\section{ACKNOWLEDGMENTS}

We are grateful to T. Fründ for technical assistance. We thank also T. Dinse for supplying the mutant $\mathrm{BH} \triangle g a c A$, A. Hermann for the mutants BHexaStd, BhelmStd, and BhgacAtd, as well as B. Bischof for the mutant BHexaS, all members of the Department of Microbe-Plant Interactions, University of Bremen. This work was supported by the University of Bremen and by a grant from the German Research Foundation to B. Reinhold-Hurek (RE 756/14-2).

\section{LITERATURE CITED}

Agarwal, S., and Grover, A. 2006. Molecular biology, biotechnology and genomics of flooding-associated low $\mathrm{O}_{2}$ stress response in plants. Crit. Rev. Plant Sci. 25:1-21.

Altschul, S. F., Madden, T. L., Schäffer, A. A., Zhang, J., Zhang, Z., Miller, W., and Lipman, D. J. 1997. Gapped BLAST and PSI-BLAST: A new generation of protein database search programs. Nucleic Acids Res. 25: 3389-3402.

Aravind, L., and Ponting, C. P. 1999. The cytoplasmic helical linker domain of receptor histidine kinase and methyl-accepting proteins is common to many prokaryotic signalling proteins. FEMS Microbiol. Lett. 176:111-116.

Bendtsen, J. D., Nielsen, H., von Heijne, G., and Brunak, S. 2004. Improved prediction of signal peptides: SignalP 3.0. J. Mol. Biol. 340:783-795.

Böhm, M., Hurek, T., and Reinhold-Hurek, B. 2007. Twitching motility is essential for endophytic rice colonization by the $\mathrm{N}_{2}$-fixing endophyte Azoarcus sp. strain BH72. Mol. Plant-Microbe Interact. 20:526-533.

Buschart, A., Sachs, S., Chen, X., Herglotz, J., Krause, A., and ReinholdHurek, B. 2012. Flagella mediate endophytic competence rather than act as MAMPS in rice-Azoarcus sp. strain BH72 interactions. Mol. PlantMicrobe Interact. 25:191-199.

Chelius, M. K., and Triplett, E. W. 2001. The diversity of archaea and bacteria in association with the roots of Zea mays L. Microb. Ecol. 41: 252-263.

Chen, M. H., Sheu, S. Y., James, E. K., Young, C. C., and Chen, W. M. 2013. Azoarcus olearius sp. nov., a nitrogen-fixing bacterium isolated from oil-contaminated soil. Int. J. Syst. Evol. Microbiol. 63: 3755-3761

Dörr, J., Hurek, T., and Reinhold-Hurek, B. 1998. Type IV pili are involved in plant-microbe and fungus-microbe interactions. Mol. Microbiol. 30: 7-17.

Egener, T., Hurek, T., and Reinhold-Hurek, B. 1999. Endophytic expression of nif genes of Azoarcus sp. strain BH72 in rice roots. Mol. PlantMicrobe Interact. 12:813-819.

Faoro, H., Menegazzo, R. R., Battistoni, F., Gyaneshwar, P., do Amaral, F. P., Taulé, C., Rausch, S., Gonçalves Galvão, P., de Los Santos, C., Mitra, S., Heijo, G., Sheu, S.-Y., Chen, W.-M., Mareque, C., Tadra-Sfeir, M. Z., Baldani, J. I., Maluk, M., Guimarães, A. P., Stacey, G., de Souza, E. M., Pedrosa, F. O., Cruz, L. M., and James, E. K. 2017. The oilcontaminated soil diazotroph Azoarcus olearius DQS- $4^{\mathrm{T}}$ is genetically and phenotypically similar to the model grass endophyte Azoarcus sp. BH72. Environ. Microbiol. Rep. 9:223-238.

Finn, R. D., Bateman, A., Clements, J., Coggill, P., Eberhardt, R. Y., Eddy, S. R., Heger, A., Hetherington, K., Holm, L., Mistry, J., Sonnhammer, E. L., Tate, J., and Punta, M. 2014. Pfam: The protein families database. Nucleic Acids Res. 42:D222-D230.

Gliese, N., Khodaverdi, V., Schobert, M., and Görisch, H. 2004. AgmR controls transcription of a regulon with several operons essential for ethanol oxidation in Pseudomonas aeruginosa ATCC 17933. Microbiology 150:1851-1857.

Haft, D. H., Selengut, J. D., Richter, R. A., Harkins, D., Basu, M. K., and Beck, E. 2013. TIGRFAMs and genome properties in 2013. Nucleic Acids Res. 41:D387-D395.

Hauberg-Lotte, L., Klingenberg, H., Scharf, C., Böhm, M., Plessl, J., Friedrich, F., Völker, U., Becker, A., and Reinhold-Hurek, B. 2012. Environmental factors affecting the expression of pilAB as well as the proteome and transcriptome of the grass endophyte Azoarcus sp. strain BH72. PLoS One 7:e30421.

Hurek, T., Burggraf, S., Woese, C. R., and Reinhold-Hurek, B. 1993. 16S rRNA-targeted polymerase chain reaction and oligonucleotide hybridization to screen for Azoarcus spp., grass-associated diazotrophs. Appl. Environ. Microbiol. 59:3816-3824.

Hurek, T., Reinhold, B., Fendrik, I., and Niemann, E. G. 1987. Root-zonespecific oxygen tolerance of Azospirillum spp. and diazotrophic rods closely associated with Kallar grass. Appl. Environ. Microbiol. 53: 163-169.

Hurek, T., Reinhold-Hurek, B., Van Montagu, M., and Kellenberger, E. 1994. Root colonization and systemic spreading of Azoarcus sp. strain BH72 in grasses. J. Bacteriol. 176:1913-1923.

Jefferson, R. A., Burgess, S. M., and Hirsh, D. 1986. beta-Glucuronidase from Escherichia coli as a gene-fusion marker. Proc. Natl. Acad. Sci. U.S.A. 83:8447-8451.

Kirchner, O., and Tauch, A. 2003. Tools for genetic engineering in the amino acid-producing bacterium Corynebacterium glutamicum. J. Biotechnol. 104:287-299.

Krause, A., Bischoff, B., Miché, L., Battistoni, F., and Reinhold-Hurek, B. 2011. Exploring the function of alcohol dehydrogenases during the endophytic life of Azoarcus sp. strain BH72. Mol. Plant-Microbe Interact. 24:1325-1332

Krause, A., Ramakumar, A., Bartels, D., Battistoni, F., Bekel, T., Boch, J., Böhm, M., Friedrich, F., Hurek, T., Krause, L., Linke, B., McHardy, A. C., Sarkar, A., Schneiker, S., Syed, A. A., Thauer, R., Vorhölter, F. J., Weidner, S., Pühler, A., Reinhold-Hurek, B., Kaiser, O., and Goesmann, A. 2006. Complete genome of the mutualistic, N2-fixing grass endophyte Azoarcus sp. strain BH72. Nat. Biotechnol. 24:1385-1391.

Krogh, A., Larsson, B., von Heijne, G., and Sonnhammer, E. L. 2001 Predicting transmembrane protein topology with a hidden Markov model: Application to complete genomes. J. Mol. Biol. 305:567-580.

Lane, D. J. 1991. 16S/23S rRNA sequencing. Pages $115-175$ in: Nucleic acids techniques in bacterial systematics. Stackebrandt, E., and Goodfellow, M., eds. John Wiley and Sons, Chichester, United Kingdom.

Marchler-Bauer, A., Lu, S., Anderson, J. B., Chitsaz, F., Derbyshire, M. K., DeWeese-Scott, C., Fong, J. H., Geer, L. Y., Geer, R. C., Gonzales, N. R., Gwadz, M., Hurwitz, D. I., Jackson, J. D., Ke, Z., Lanczycki, C. J., Lu, F., Marchler, G. H., Mullokandov, M., Omelchenko, M. V., Robertson, C. L., Song, J. S., Thanki, N., Yamashita, R. A., Zhang, D., Zhang, N., Zheng, C., and Bryant, S. H. 2011. CDD: A conserved domain database 
for the functional annotation of proteins. Nucleic Acids Res. 39: D225-D229.

Medema, M. H., Takano, E., and Breitling, R. 2013. Detecting sequence homology at the gene cluster level with MultiGeneBlast. Mol. Biol. Evol. 30:1218-1223.

Mern, D. S., Ha, S. W., Khodaverdi, V., Gliese, N., and Görisch, H. 2010. A complex regulatory network controls aerobic ethanol oxidation in Pseudomonas aeruginosa: Indication of four levels of sensor kinases and response regulators. Microbiology 156:1505-1516.

Mustroph, A., and Albrecht, G. 2003. Tolerance of crop plants to oxygen deficiency stress: Fermentative activity and photosynthetic capacity of entire seedlings under hypoxia and anoxia. Physiol. Plant. 117:508-520.

Oberto, J. 2013. SyntTax: A web server linking synteny to prokaryotic taxonomy. BMC Bioinformatics 14:4.

Ortet, P., Whitworth, D. E., Santaella, C., Achouak, W., and Barakat, M. 2015. P2CS: Updates of the prokaryotic two-component systems database. Nucleic Acids Res. 43:D536-D541.

Prentki, P., and Krisch, H. M. 1984. In vitro insertional mutagenesis with a selectable DNA fragment. Gene 29:303-313.

Promden, W., Vangnai, A. S., Toyama, H., Matsushita, K., and Pongsawasdi, P. 2009. Analysis of the promoter activities of the genes encoding three quinoprotein alcohol dehydrogenases in Pseudomonas putida HK5. Microbiology 155:594-603.

Reinhold, B., Hurek, T., Niemann, E.-G., and Fendrik, I. 1986. Close association of azospirillum and diazotrophic rods with different root zones of Kallar grass. Appl. Environ. Microbiol. 52:520-526.

Reinhold-Hurek, B., and Hurek, T. 2011. Living inside plants: Bacterial endophytes. Curr. Opin. Plant Biol. 14:435-443.

Reinhold-Hurek, B., Hurek, T., Gillis, M., Hoste, B., Vancanneyt, M., Kersters, K., and De Ley, J. 1993. Azoarcus gen. nov., nitrogen-fixing proteobacteria associated with roots of Kallar grass (Leptochloa fusca (L.) Kunth) and description of two species Azoarcus indigens sp. nov. and Azoarcus communis sp. nov. Int. J. Syst. Bacteriol. 43:574-584.

Reinhold-Hurek, B., Maes, T., Gemmer, S., Van Montagu, M., and Hurek, T. 2006. An endoglucanase is involved in infection of rice roots by the not-cellulose-metabolizing endophyte Azoarcus sp. strain BH72. Mol. Plant-Microbe Interact. 19:181-188.

Sambrook, J., Fritsch, E. F., and Maniatis, T. 1989. Molecular Cloning. A Laboratory Manual. Cold Spring Harbor Laboratory Press, Cold Spring Harbor, NT, U.S.A.

Sarkar, A., Marszalkowska, M., Schäfer, M., Pees, T., Klingenberg, H., Macht, F., and Reinhold-Hurek, B. 2017. Global expression analysis of the response to microaerobiosis reveals an important cue for endophytic establishment of Azoarcus sp. BH72. Environ. Microbiol. 19:198-217.

Sarkar, A., and Reinhold-Hurek, B. 2014. Transcriptional profiling of nitrogen fixation and the role of NifA in the diazotrophic endophyte Azoarcus sp. strain BH72. PLoS One 9:e86527.

Schäfer, A., Tauch, A., Jäger, W., Kalinowski, J., Thierbach, G., and Pühler, A. 1994. Small mobilizable multi-purpose cloning vectors derived from the Escherichia coli plasmids pK18 and pK19: Selection of defined deletions in the chromosome of Corynebacterium glutamicum. Gene 145:69-73.

Schobert, M., and Görisch, H. 2001. A soluble two-component regulatory system controls expression of quinoprotein ethanol dehydrogenase (QEDH) but not expression of cytochrome $c_{550}$ of the ethanol-oxidation system in Pseudomonas aeruginosa. Microbiology 147:363-372.

Shidore, T., Dinse, T., Öhrlein, J., Becker, A., and Reinhold-Hurek, B. 2012. Transcriptomic analysis of responses to exudates reveal genes required for rhizosphere competence of the endophyte Azoarcus sp. strain BH72. Environ. Microbiol. 14:2775-2787.

Springer, A. L., Morris, C. J., and Lidstrom, M. E. 1997. Molecular analysis of $m x b D$ and $m x b M$, a putative sensor-regulator pair required for oxidation of methanol in Methylobacterium extorquens AM1. Microbiology 143: 1737-1744.

Tribelli, P. M., Solar Venero, E. C., Ricardi, M. M., Gómez-Lozano, M., Raiger Iustman, L. J., Molin, S., and López, N. I. 2015. Novel essential role of ethanol oxidation genes at low temperature revealed by transcriptome analysis in the antarctic bacterium Pseudomonas extremaustralis. PLoS One 10:e0145353. 